\title{
An approach for SLAR images denoising based on removing regions with low visual quality for oil spill detection
}

Beatriz Alacid*a, Pablo Gil ${ }^{\mathrm{b}}$

${ }^{a}$ Computer Science Research Institute, University of Alicante, Alicante, Spain

${ }^{b}$ Dept. of Physics, Systems Engineering and Signal Theory, University of Alicante, Alicante, Spain

\begin{abstract}
This paper presents an approach to remove SLAR (Side-Looking Airborne Radar) image regions with low visual quality to be used for an automatic detection of oil slicks on a board system. This approach is focused on the detection and labelling of SLAR image regions caused by a poor acquisition from two antennas located on both sides of an aircraft. Thereby, the method distinguishes ineligible regions which are not suitable to be used on the steps of an automatic detection process of oil slicks because they have a high probability of causing false positive results in the detection process. To do this, the method uses a hybrid approach based on edge-based segmentation aided by Gabor filters for texture detection combined with a search algorithm of significant grey-level changes for fitting the boundary lines in each of all the bad regions. Afterwards, a statistical analysis is done to label the set of pixels which should be used for recognition of oil slicks. The results show a successful detection of the ineligible regions and consequently how the image is partitioned in sub-regions of interest in terms of detecting the oil slicks, improving the accuracy and reliability of the oil slick detection.
\end{abstract}

Keywords: SLAR, oil spill, segmentation, detection, denoising, saliency map.

\section{INTRODUCTION}

Nowadays the use of Synthetic Aperture Radar (SAR) is very common in both maritime and land field. This radar is incorporated in satellites at high altitude and its function is to process the captured information from the radar antenna, by means of building of a virtual scanning from multiple real scanning [1]. The Side-Looking Airborne Radar (SLAR) is based on SAR. The main difference between SAR radar and SLAR is that the latter is incorporated in manned aircraft.

On land, SAR technology has been used for geologic studies, natural hazards like flooding and earthquake [2, 3, 4]. In [4] a method is shown to classify supervised areas with flooding hazard, performing an analysis of SAR images. Others are related to forestry studies [5] where SAR images are combined with information from other radars to analyze forest areas, defining their vegetation composition. Concerning maritime studies, in this case, these radars are used for ship detection [6], marine biology studies as well as sea ice [7] and phytoplankton [8] formation.

One aspect to take into account when images are captured with both SAR and SLAR technologies, is the noise, which complicates the image interpretation and the detection performance. In works such as [9], filters to reduce speckle noise (multiplicative noise that results from random fluctuations of signals when they are reflected on a surface) are shown. These filters anticipate that the image data fulfil approximately a statistical model and its objective is to reduce the speckle preserving the image details. Victor S. Frost et al. in [10] proposed a filter method to smooth the noise of SAR images. This is a filter based on Mean Squared Error (MSE) to smooth images using estimated local parameters in homogeneous areas. In [11] a technique for attenuating the noise is proposed. This is done using classic statistical filters and a sizeadaptive window to provide effective noise reduction and edge preservation.

In the same vein, other authors in [12] explained a method which combines both the wavelet Bayesian and the Markovrandom technique. Fabrizio Argenti et al. in [13] presented a method based on MMSE (Minimum mean square error) using the undecimated wavelet domain by means of an adaptive re-scaling of the detail coefficients, whose amplitude is divided by the variance ratio of the noisy coefficient to the noise-free one. In [14] a Bayesian algorithm is proposed which reduces noise in SAR images while preserving structural features and textural information of the scenes. To do that, a sub-band decomposition of images is carried out with logarithmic transformations and modelling with precision by alpha-stable distribution.

Birgir Bjorn Saevarsson et al. [15] present a strategy to eliminate the speckle noise using the combination of two algorithms. First, the CFA (Combined Filtering Algorithm) is based on constrained optimization problem, both in the 
wavelet and curvelet domains. The second method is called the Adaptive Combined Method (ACM), which uses the wavelet transform to denoise homogeneous areas and the curvelet transform to denoise areas with edges. The authors in [16] proposed a spatially adaptive wavelet-based Bayesian method to denoise. To do this, the wavelet coefficients of the logarithmically transformed reflectance and speckle noise are modelled using zero-location Cauchy and zero-mean Gaussian distributions, respectively. These prior distributions are then exploited to develop a Bayesian minimum mean absolute error estimator as well as a maximum a posteriori estimator.

In [17] L. Gagnon and A. Jouan presented a comparative study between the WCS (Wavelet Coefficient Shrinkage) and various standard noise suppression filters (Lee, Kuan, Frost, Kalman, etc.). WCS filter is based on the use of SD (Symmetric Daubechies) wavelets, which are obtained from MRA (Multi-Resolution Analysis).

For the oil spill detection, various techniques are used as extracting features on an image, segmentation techniques and regions labelling, artificial intelligence for recognition and classification, etc. In processes like [18] the oil detection is based on the search of dark regions on the image. In order to do this, a segmentation by adaptive threshold is carried out. From these regions some characteristics are extracted from which a classification of regions between oil and look-a-likes is carried out. Lena Chang et al. in [19] propose a segmentation method that combines the preservation of the moment, and regions clustering. Other proposals like $[20,21]$ are based on the use of an algorithm with adaptive thresholding depending on the information the SAR sensor provides and the wind estimation on the marine surface. In [22], an algorithm is proposed by the characterization of the oil spill shape which determines whether it is oil spill.

The aforementioned studies indicate that there are a great variety of works focused using images with SAR technology for oil spill detection. However, there is a lack of research using SLAR images for oil spill detection mainly due to the noise zones generated by the SLAR sensor setting, its location and the aircraft trajectory. Therefore, a processing method is proposed with the objective of optimizing the detection of possible oil spills in SLAR images.

This paper has been organized in the following way: firstly in section two, a method for the noise detection in SLAR images (blind zones and aircraft turns) is proposed. In section three, an approach is proposed for the detection of possible oil spills in SLAR images, based on the Fourier Transform and the spectral residue. Finally, in section 4 and 5, the results obtained are presented for each proposed method and the conclusions based on these results.

\section{DENOISING}

The SLAR images generated by sensor scans, tend to indicate noise areas as a consequence of sensor signal failures (blind zone), changes of direction and turns from surveillance aircraft. Therefore, these areas are distinguished by registering important measurement errors in the image, making this one unsuitable for applying image process techniques on them. They have to be identified and delimited to prevent them considering both analysis and automatic detection processes which may alter the results of oil spill detection (avoiding no desirable detections like false positives).

This method's main objective is the identification in order to delimitate the area with non-processable spots in images (turns and blind zones) so as to set the parameters in every oil spill detection process, and thus prevent false positives and false measurements. This approach takes into consideration the fact that the location of blind zone and the aircraft turns are unknown because location changes depend on manoeuvres and flight conditions of the aircraft. In this way, a maximization of optimal image processing is obtained.

Due to the assembly and set up of the SLAR sensor on the aircraft, the blind zone which maps the flight path, is identified by its contrasting shade compared to the rest of the image. The turn areas (produced by warping inclination) are identified by the change of contrast in regions, perpendicular to the direction of the flight path. The SLAR blind zone is visually delimited by two virtual vertical lines, whereas turns are delimited by straight horizontal lines perpendicular to the blind zone.

In order to detect SLAR blind zones and aircraft turns, we have developed and introduced the following method (Figure 1). First the image is segmented by textures using Gabor filters [23] by means of, assigning a frequency $f$ and direction $d$. The Canny algorithm is applied to the output image, in order to obtain those pixels where there is a change of intensity compared to their neighbors. It is based on directional gradients [24]. Afterwards, the Hough transform is used [25] to calculate the straight lines in image that delimit those areas (equation 1, where $\rho$ is the perpendicular distance from the origin to the straight line and $\theta$ is the angle formed by the perpendicular line and the horizontal axis). This algorithm 
provides the set of the straight lines that bring nearer points with same intensity gradient. At this point, the algorithm is splits in two pipelines, based on the target detection, namely, the blind zone or the aircraft turns. In section 2.1, the distinctives features of the detection of the blind zone and of the aircraft turns method are described.
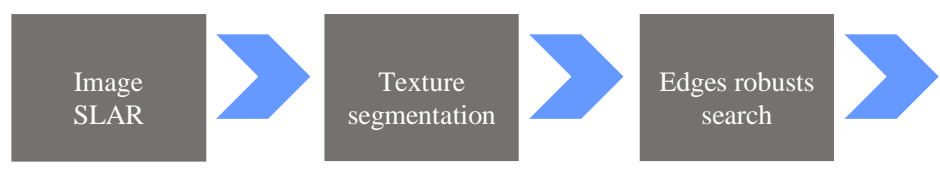

Extracting of the straights
Parameteri-

zation blind

zone

SLAR

Figure 1. Method diagram.

\subsection{Detection of both aircraft blind and turning zones}

As discussed above, the SLAR images used in this paper, the blind zone is delimited by vertical straight lines (see section 2), a fact determined by the direction used in the Gabor filters, in this case $90^{\circ}$. In this way, a vertical texture segmentation is carried out. Another peculiarity of the blind zone detection occurs when applying the Hough transform, to extract only those straight lines oriented at $90^{\circ}$. From the set of the vertical straight lines oriented at $90^{\circ}$, only a pair of them are extracted, that is, those with longer distance between themselves.

For the aircraft turns detection, it should be taken into consideration that these are determined by horizontal straight lines. In this case, the direction is $180^{\circ}$, when applying the Gabor filters for the image segmentation. Next, when applying the Hough transform, only the horizontal straight lines oriented at $180^{\circ}$ will be considered. This way, every turn will be delimited by only a pair of horizontal straight lines oriented at $180^{\circ}$.

Figure 2 shows an example of the Hough transform, specifically a part of the result of the voting matrix obtained for the selected fragments of the image. In fragment 1 , the most voted box is the corresponding to $\theta=180^{\circ}$. Additionally, fragment 2 , the most voted box is $\theta=90^{\circ}$.

$$
\rho=x \cdot \cos \theta+y \cdot \sin \theta
$$

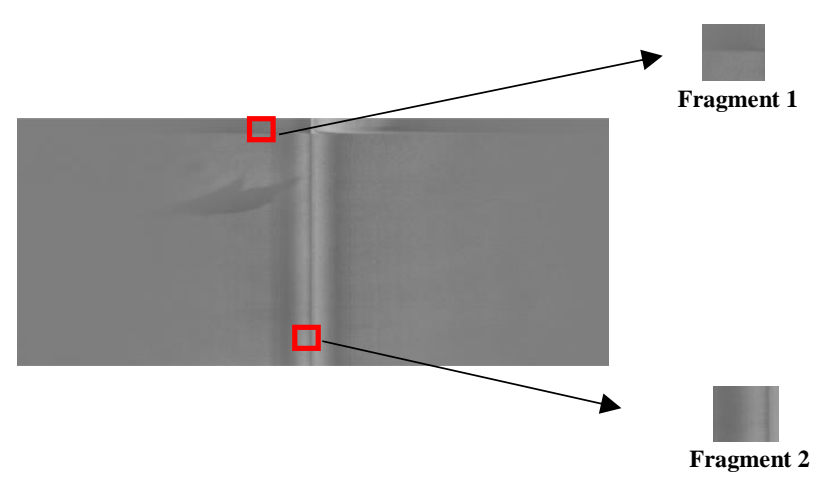

\begin{tabular}{|c|c|c|c|c|c|c|}
\hline & \multicolumn{7}{|c|}{$\theta$} \\
\hline \multirow{4}{*}{$\rho$} & 0 & 0 & 0 & 0 & 0 & 0 \\
\cline { 2 - 7 } & 0 & 0 & 0 & 0 & 0 & 0 \\
\cline { 2 - 7 } & 0 & 0 & 0 & 0 & 0 & 7 \\
\cline { 2 - 7 } & 0 & 0 & 0 & 0 & 0 & 1 \\
\cline { 2 - 7 } & 0 & 2 & 0 & 0 & 0 & 0 \\
\cline { 2 - 7 } & 0 & 2 & 0 & 0 & 0 & 0 \\
\hline
\end{tabular}

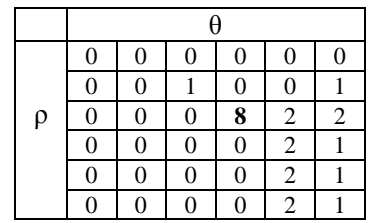

Figure 2. Hough Transform example.

It should also be noted that both the number of turns on the image and their position are unknown. Therefore, a method based on statistical analysis has been introduced, in order to identify both the position of the centroids for each turn and 
the number of these on the image. This method is based on the k-means algorithm [26] (see equation 2, where $\mu$ is the mean of points in $S_{i}$, being $S_{i}$ the set of points that define the straight lines obtained for each turn). The implemented method determines the number of turns made. In order to do that, the k-means algorithm is executed with different cluster values (where each cluster defines a limit that divides two regions, in other words, a detected straight line). Initially, $\mathrm{k}=1$ initial centroid is set, the straight lines are grouped depending on the distance between the straight line and the selected centroid, so that each straight line is part of the nearest cluster. Afterwards, the previous steps are repeated for the different $\mathrm{k}$ values. Once they are executed, the optimal number of clusters is established, being this the one that minimizes the variance between clusters. This way, the number of turns on the image is determined by the number of obtained clusters when applying the algorithm. Each turn will be delimited by two straight lines, which are determined by the distance from this one to the centroid of the turn. The highest level is the straight line which is situated furthest from the centroid and with the lowest ' $y$ ' axis. In addition, the lowest level is the straight line which is situated furthest from the turn centroid (Figure 3, where the red lines are group of lines found for every turn, and the blue lines are the highest and the lowest levels that delimit the turn).

$$
\arg \min \sum_{i=1}^{k} \sum_{x_{j} \in S_{i}}\left\|x_{j}-\mu_{i}\right\|^{2}
$$

$$
\mathrm{S}=\left\{\mathrm{S}_{1}, \mathrm{~S}_{2}, \ldots, \mathrm{S}_{\mathrm{k}}\right\}
$$

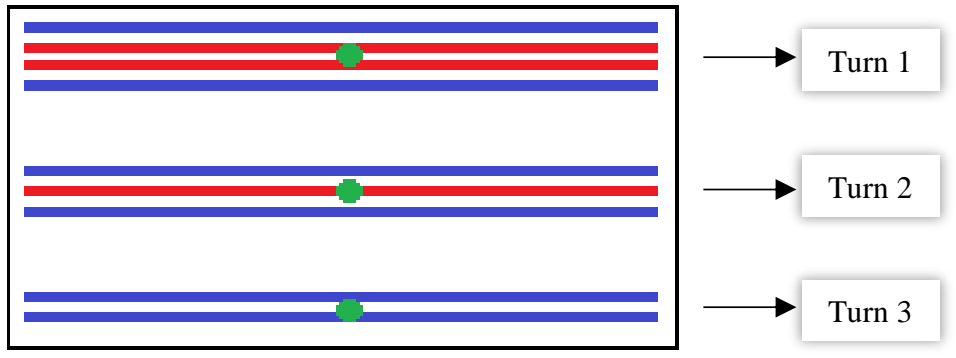

Figure 3. Example schematic of the set of turns detected for a SLAR image.

\subsection{Image decomposition on sub-images}

The next step is to decompose the image into sub-images where the regions, which represent with both the turns and the blind zones, are eliminated. As a result, a set of sub-images is obtained, where the oil spills detection is improved and where the false positives due to noised areas are prevented. In order to accomplish this, first the original image is divided into two sub-images. One sub-image would be the left hand side of the original image, this means from $\rho=0$ to $\rho_{1}-1$. The other one would be from $\rho_{2}+1$ to $\rho=$ total_width.

Once the image has been vertically divided into two sub-images, the horizontal division follows depending on the found turns. As indicated in the turn detection results, every turn is represented by a set of horizontal straight lines. Thus the demarcation of the turn is determined by two straight lines: the line further from the middle point of the turn (value on position obtained with k-means) and the line further from this previous line. This means that the set of sub-images is determined by the position of every turn where $\mathrm{g}=\left\{\mathrm{g}_{1}, \mathrm{~g}_{2}, \ldots, \mathrm{g}_{\mathrm{n}}\right\}$ is the set of turns detected on the image $\mathrm{I}$ and $\mathrm{g}_{\mathrm{n}}=\left\{\rho_{\mathrm{gn} 1}\right.$, $\rho_{\mathrm{gn} 2}$. The first sub-image starts at $\rho=0$ and ends at $\rho_{\mathrm{g} 11}-1$, being this the $\rho$ value for the last straight line before the beginning of the first turn. The next sub-image starts at $\rho_{\mathrm{g} 12}+1$ and ends at $\rho_{\mathrm{g} 21}-1$. Usually, every sub-image starts at $\rho_{\mathrm{gn} 2}+1$ and ends at $\rho_{\mathrm{gn} 1}-1$. Except for the last sub-image, which starts at $\rho_{\mathrm{gn} 2}+1$ and will ends at $\rho=$ total_height. Figure 4 shows the result of the division of the image from Figure 3. 


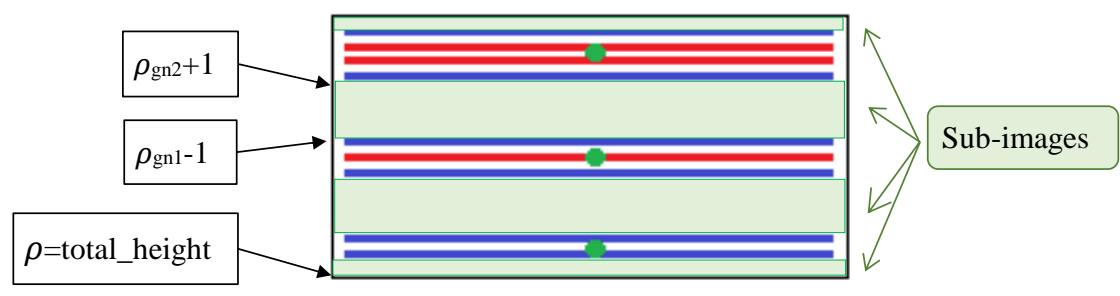

Figure 4. Set of sub-images obtained from the example shown in Figure 3.

\section{EXTRACTION OF OIL SPILL REGION}

\subsection{Sub-image enhancing for differentiating oil spills}

After decomposing the ROIs image, a mitigation method is applied to the sub-images which have noise and lack of homogeneity, even when no spilled zone exists. In some cases, the sub-images may have zones which look like an oil spill but they are false positives. This is due to the sensor acquisition process and the environmental conditions.

In this denoising method, first the noise from the acquisition process is eliminated applying the Gaussian filter. Then, the shape and values from the histogram are analyzed, and an equalization process is performed to improve the visual image. The process aims to saturate the $2 \%$ of values to 0 or 255 . This way, a higher contrast is obtained, eliminating atypical low intensity or high intensity values that saturate at minimum or maximum values as appropriate. Afterwards, the result is processed to reduce the intensity level and to eliminate borders of isolated regions, saturating the pixels that are much brighter than their 8-neighboor environment. This makes possible the size reduction and the compression of the regions that, even if they have similar intensity values to spill represented regions, are remaining noised regions from the acquisition process. Unfortunately, these regions remain because the previous filters were unable to totally eliminate them. So, at this stage, these types of non-representative regions are blurred or smoothed in the image for future detection and processing stages, ensuring that they are not easily confused with regions that do represent oil spills.

Figure 5 shows the processing of a small ROI obtained from a sub-images (Figure 8.b).

a)

\begin{tabular}{|r|r|r|r|r|r|r|r|r|r|r|r|}
\hline 105 & 106 & 102 & 104 & 109 & 107 & 104 & 103 & 105 & 100 & 104 & 107 \\
\hline 99 & 102 & 100 & 100 & 96 & 102 & 98 & 109 & 103 & 104 & 106 & 102 \\
\hline 102 & 98 & 100 & 108 & 102 & 97 & 104 & 98 & 105 & 99 & 103 & 103 \\
\hline 96 & 99 & 109 & 100 & 102 & 102 & 102 & 99 & 99 & 105 & 105 & 106 \\
\hline 99 & 102 & 102 & 99 & 119 & 102 & 106 & 102 & 105 & 102 & 103 & 105 \\
\hline 111 & 102 & 105 & 103 & 104 & 102 & 109 & 107 & 109 & 104 & 106 & 107 \\
\hline 112 & 122 & 117 & 113 & 115 & 109 & 111 & 113 & 117 & 123 & 109 & 120 \\
\hline 126 & 135 & 123 & 125 & 124 & 124 & 121 & 122 & 122 & 124 & 126 & 122 \\
\hline 124 & 131 & 131 & 139 & 131 & 131 & 129 & 128 & 133 & 124 & 126 & 132
\end{tabular}

b)

\begin{tabular}{|l|l|l|l|l|l|l|l|l|l|l|l|}
\hline 225 & 230 & 209 & 214 & 230 & 230 & 214 & 214 & 220 & 204 & 214 & 225 \\
\hline 194 & 204 & 199 & 199 & 192 & 199 & 194 & 225 & 214 & 209 & 220 & 209 \\
\hline 194 & 192 & 199 & 220 & 204 & 192 & 204 & 194 & 209 & 199 & 209 & 209 \\
\hline 192 & 194 & 225 & 204 & 209 & 204 & 204 & 194 & 199 & 214 & 220 & 220 \\
\hline 194 & 204 & 209 & 204 & 255 & 214 & 220 & 209 & 214 & 209 & 214 & 225 \\
\hline 235 & 220 & 225 & 214 & 225 & 214 & 235 & 235 & 240 & 225 & 225 & 240 \\
\hline 255 & 255 & 255 & 255 & 255 & 250 & 255 & 255 & 255 & 255 & 255 & 255 \\
\hline 255 & 255 & 255 & 255 & 255 & 255 & 255 & 255 & 255 & 255 & 255 & 255 \\
\hline 255 & 255 & 255 & 255 & 255 & 255 & 255 & 255 & 255 & 255 & 255 & 255 \\
\hline
\end{tabular}

Figure 5. a) An example of an input image and b) resulting image after applying the enhancing method.

\subsection{Model of spectral residual for location of oil slicks}

In order to detect the candidate oil spills, a method based on the Fourier transform [27] and the resulting spectral residue [28] has been implemented. Afterwards, a saliency map from the sub-images on section 2 is obtained. Firstly, the frequency of the images is analyzed and the spectral residues are extracted. The saliency map is obtained by transforming the spectral residue from the frequency domain into the time domain. In Figure 6, a diagram with the different phases of this method is shown. This one is based on three main stages: Fourier transform, spectral residue calculation and inverse Fourier transform. 
Figure 6. Diagram of the oil spills detection method.

Firstly, the frequency $\mathrm{f}$ of the image is obtained by calculating the Fourier transform (see equation 1 , where ' $\mathrm{n}$ ' is the number of samples taken in time $[0, \mathrm{~T}])$.

$$
\begin{gathered}
F(x)=\sum_{j=1}^{n} X(j) \cdot W_{n}^{(j-1)(x-1)} \\
X(j)=\frac{1}{N} \sum_{j=1}^{N} x(k) \omega_{N}^{-(j-1)(k-1)} \\
W_{n}=e^{(-2 \pi i) / n}
\end{gathered}
$$

Where $f=F(I(x))$, being $I(x)$ the initial image. The amplitude matrix is determined as $A(f)=a b s(f)$ and the phases matrix as $P(f)=a n g l e(f)$. The first matrix refers to the information related to the intensity values in the image, and the second matrix refers to the information related to the pixels position. The next step is to obtain the spectral residue R(f) from each one of the sub-images from the set of images which potentially have oil spills. This is defined by equation 4 , where $\mathrm{L}(\mathrm{f})$ is the amplitude matrix logarithm and $\mathrm{h}(\mathrm{f})$ is a mean filter whose mask has a $3 \times 3$ size.

$$
\begin{gathered}
R(f)=L(f)-h(f) x L(f) \\
L(f)=\log (A(f)) \\
h(f)=\frac{1}{3 \times 3}\left(\begin{array}{lll}
1 & 1 & 1 \\
1 & 1 & 1 \\
1 & 1 & 1
\end{array}\right)
\end{gathered}
$$

Once the spectral residue is calculated, $\mathrm{R}(\mathrm{f})$ and the phases matrix, $\mathrm{P}(\mathrm{f})$, the corresponding saliency map is obtained with the inverse Fourier transform. This provides a compressed representation of an image, shown on equation 7.

$$
\begin{aligned}
& S(x)=F^{-1}[\exp (R(f)+P(f))]^{2} \\
& F^{-1}(j)=\frac{1}{n} \sum_{k=1}^{n} F(x) \cdot W_{n}^{-(j-1)(x-1)}
\end{aligned}
$$

Finally, the saliency map is binarized, obtaining an image pixel classification as background or an objects. In order to do that, a grey threshold needs to be established, in a way that all the pixels with a lower grey level are classified as objects, and those whose grey level is above the selected threshold are classified as background. Once the binarized saliency map is realized, the geometric centroid for each of the detected objects is calculated.

\section{EXPERIMENTAL RESULTS AND ANALYSIS}

The following results were obtained after applying the method for the detection of the blind zone and the aircraft turns on a SLAR image as described in section 2. The aircraft's environmental and flight conditions when the images were taken should be taken into consideration. It is worth noting that the information captured with SLAR sensors is unreliable under low wind conditions. For the following images, the altitude is approximately 4500 feet, the flight speed about $200 \mathrm{Kn}$ and wind speed is between $6 \mathrm{Kn}$ and $25 \mathrm{Kn}$. 
Figures 7.a and 7.d are to examples for input SLAR images, from which the tests have been performed. Next, Figures 7.b and 7.e show the results after applying the blind zone detection. Then, the turns detection method is applied and the results are show in Figure 7.c and 7.f.

a)

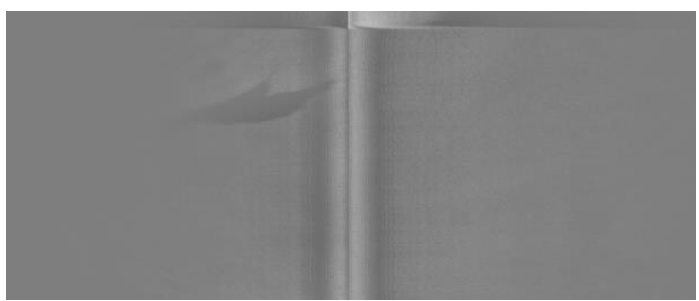

b)

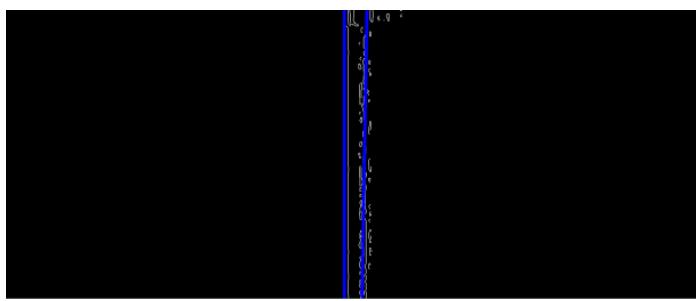

c)

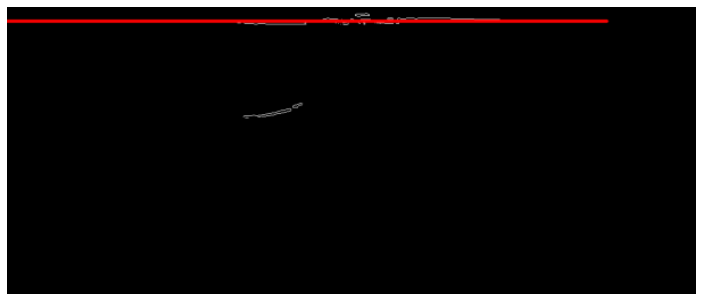

d)

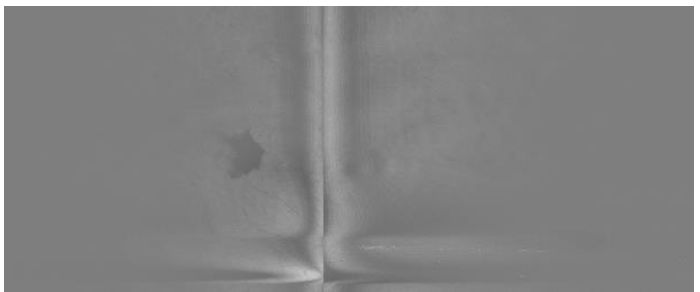

e)

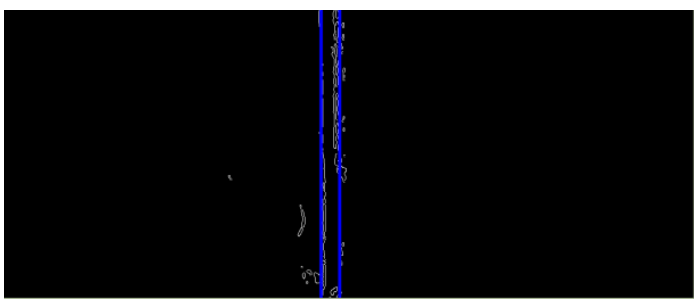

f)

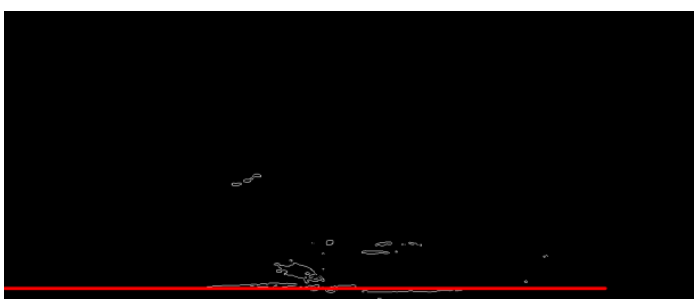

Figure 7. a) and d) Input SLAR images. b) and e) output images after the blind zone detection. c) and f) output images after the turns detection.

After detecting the different noised areas such as blind zone and the aircraft turns, a set of sub-images is obtained from the input one, eliminating the mentioned zones in those sub-images. Figures 8 and 9 show the set of sub-images obtained from the original SLAR images in Figure 7.a and Figure 7.d, respectively.

a)

b)

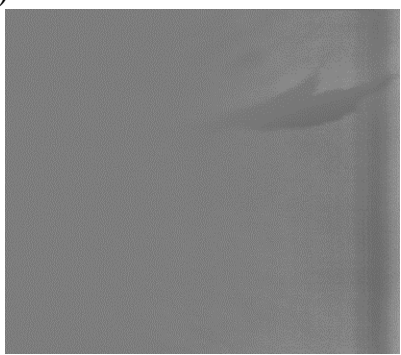

c)

d)

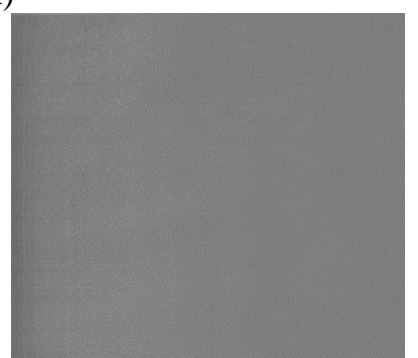

Figure 8. Set of sub-images obtained where the blind zone and the aircraft turns have been eliminated. 
a)

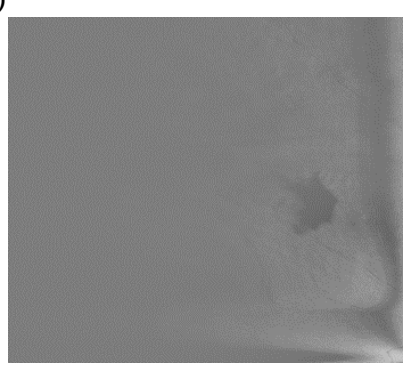

b) c)

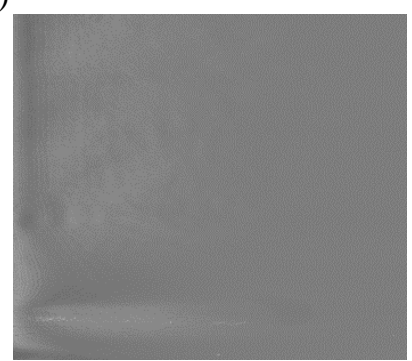

d)

Figure 9. Set of sub-images obtained where the blind zone and the aircraft turns have been eliminated.

Below, the results obtained with the method of the detection of possible zones containing oil spills are shown. A comparison of the obtained results with the input SLAR image is carried out, meaning, the image where the noise zones have not been eliminated (blind zone and aircraft turns) compared with the sub-images obtained decomposing the original image eliminating the noise areas.

Figure 10 shows the saliency map obtained from the SLAR sub-images in Figures 8 and 9, respectively.

a)

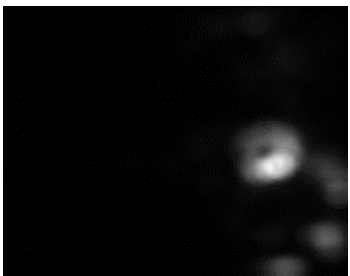

b)

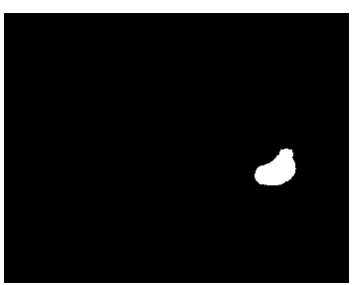

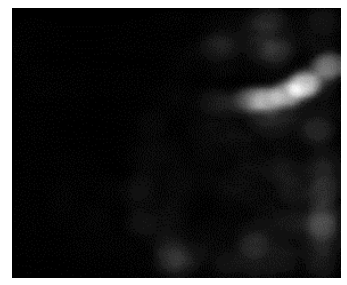

d)

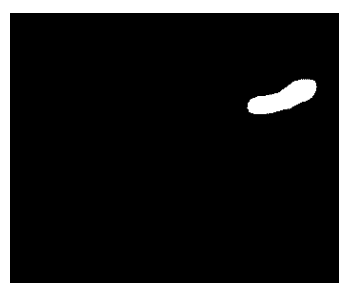

Figure 10. a) Output image by applying the detection method to Figure 9.b. b) output image from the thresholding of Figure 10.a. c) Output image by applying the detection method to Figure 8.a. d) Saliency map resulting from the binarization.of Figure 10.c.

Furthermore, the results obtained from the original images, in which the noise zones have not been eliminated, are shown in Figures 11 and 12. The resulting saliency map for both images indicates that the obtained zones do not match any oil slick. The main reason for this is that the noise areas alter the detection method performance generating false positives. 


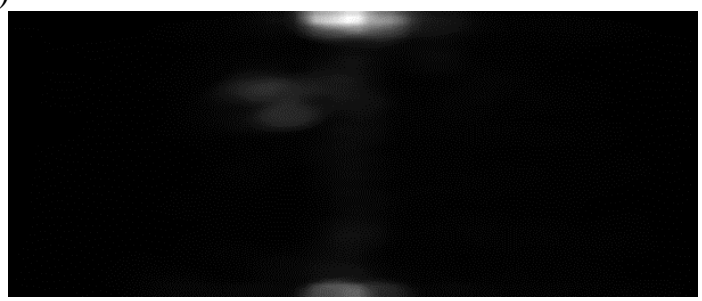

b)

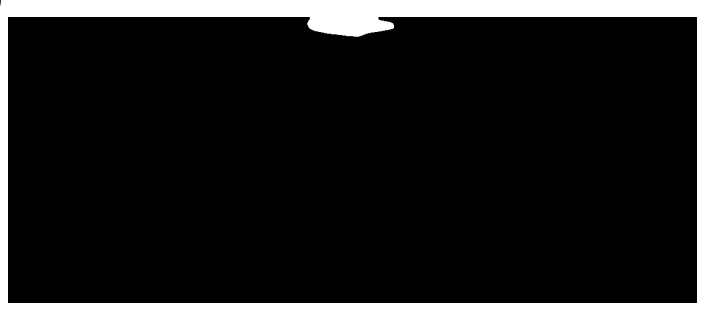

Figure 11. a) Saliency map obtained from image in Figure 7.a and b) output image after binarization.

a)

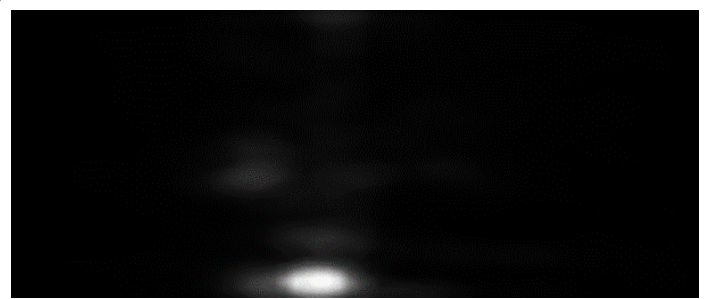

b)

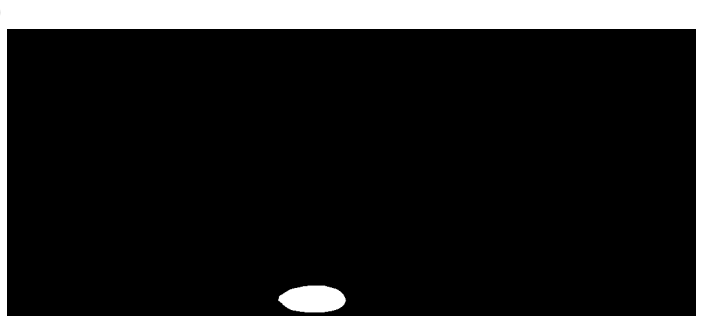

Figure 12. a) Saliency map obtained from image in Figure 7.d and b) output image after binarization.

\section{CONCLUSIONS AND FUTURE WORKS}

As mentioned above, there are a great variety of works dealing the detection of oil slicks using SAR images. However, in SLAR images there is a lack of works with the same goal, the main issues probably is the noised zones generated by the way that these type images are acquired.

In this paper was presented, on the one hand an automatic method to detect and eliminate noise in the SLAR images and on the other hand, a method to detect the possible oil spills in the images by applying a detection method based on decomposition of sub-images and spectral residual. According to the results obtained and showed in section 4, the detection of the oil slicks on SLAR images is accurate and the number of false positives obtained is decremented when the denoising method is applied in a previous stage. Nowadays, we are working for optimizing the methods presented in this paper.

Future works are focused on studying classification algorithms based on SVM (Support-Vector Machine) to classify the candidate regions obtained between oil slick and false positive.

\section{ACKNOWLEDGMENT}

This work was supported by the project (RTC-2014-1863-8) of call for collaboration challenges MINECO. We thank to INAER Helicopters S.A.U staff, which is a part of Babcock International Group.

\section{REFERENCES}

[1] Curlander, John C., Mcdonough, Robert N., [Synthetic Aperture Radar], New York, NY, USA: John Wiley \& Sons, (1991).

[2] F.J. Meyer, D.B. McAlpin, W. Gong, O. Ajadi, S. Arko, P.W. Webley, J. Dehn, "Integrating SAR and derived products into operational volcano monitoring and decision support systems", ISPRS Journal of Photogrammetry and Remote Sensing, 100, 106-117 (2015).

[3] Herrera, G., Tomás R., López-Sánchez, J., Monserrat, O., Cooksley, G., Mulas J., "Sistemas radar aplicados a la investigación de subsidencia y movimientos de ladera", Enseñanza de las Ciencias de la Tierra, 17, 1132-9157 (2009). 
[4] Avedaño-Pérez, Parra Plazas, J.A., Fredy Bayona J., "Segmentación y clasificación de imágenes SAR en zonas de inundación en Colombia, una herramienta computacional para prevención de desastres”, Revista inge @ uan, 4(8), 2438 (2014).

[5] Wolter, Peter T., Townsend, Philip A., "Multi-sensor data fusion for estimating forest species composition and abundance in northern Minnesota", ELSEVIER Journal Remote Sensing of Environment, 184, 1-14 (2016).

[6] Wang, C., Liao, M., Li, X., "Ship Detection in SAR Image Based on the Alpha-stable Distribution", Sensors, 8(8), 4948-4960 (2008).

[7] Back, D., Holt, B., Kwok, R., "Analysis of C-band Polarimetric Signatures of Arctic Lead Ice using Data from AIRSAR and RADARSAT-1", IGARSS 2008 - 2008 IEEE International Geoscience and Remote Sensing Symposium, Boston, MA, 184- 187 (2008).

[8] SvejKovsky, J., Shandley, J., "Detection of offshore plankton blooms with AVHRR and SAR imagery", International Jorunal of Remote Sensing, 22(2-3), 471-485 (2001).

[9] Bustos, O.H., Palacio, M.G., Frery, A.C., "Filtros interactivos reductores de ruido speckle en imágenes", Revista de Teledetección, 17, 61-70(2002).

[10] Frost, V. S., Stiles, J. A., Shanmugan, K. S., Holtzman, J. C., "A Model for Radar Images and Its Application to Adaptive Digital Filtering of Multiplicative Noise", IEEE Transactions on Pattern Analysis and Machine Intelligence, PAMI-4(2), 157-166 (1982).

[11] Quinones, J., Prieto, F., "Reduction of Speckle Noise by Using an Adaptive Window", Ingenierías Universidad de Medellín, 11(20), 179-190 (2012).

[12]Xie,H., Pierce, L. E., Ulaby, F. T., "SAR speckle reduction using wavelet denoising and Markov random field modeling", IEEE Transactions on Geoscience and Remote Sensing, 40(10), 2196-2212(2002).

[13] Argenti, F., Alparone, L., "Speckle removal from SAR images in the undecimated wavelet domain", IEEE Transactions on Geoscience and Remote Sensing, 40(11), 2363-2374(2002).

[14] Achim, A., Tsakalides, P., Bezerianos, A., "SAR image denoising via Bayesian wavelet shrinkage based on heavytailed modeling", IEEE Transactions on Geoscience and Remote Sensing, 41(8), 1773-1784(2003).

[15] Saevarsson, B. B., Sveinsson, J. R., Benediktsson, J. A., "Combined wavelet and curvelet denoising of SAR images", IGARSS '04. Proc. 2004 IEEE International, Anchorage, 6, 4235-4238 (2004).

[16] Bhuiyan, M. I. H., Ahmad, M. O., Swamy, M. N. S., "Spatially Adaptive Wavelet-Based Method Using the Cauchy Prior for Denoising the SAR Images", IEEE Transactions on Circuits and Systems for Video Technology, 17(4), 500507, (2007).

[17] Gagnon, L., Jouan, A., "Speckle filtering of SAR images: a comparative study between complex-wavelet-based and standard filters", Proc. SPIE 3169, 80 (1997).

[18] Singha, S., Bellerby, T. J., Trieschmann, O., "Detection and classification of oil spill and look-alike spots from SAR imagery using an Artificial Neural Network", Proc. IEEE International Geoscience and Remote Sensing Symposium, 5630-5633(2012).

[19] Özkan, C., Öztürk, C., Sunar, F., Karaboğa, D., "The Artificial Bee Colony Algorithm in Training Artificial Neural Network for Oil Spill Detection", Neural Network World, 21, 473-492 (2011).

[20] Pelizzari, S., Bioucas-Dias, J., "Oil spill segmentation of SAR images via graph cuts", 2007 IEEE International Geoscience and Remote Sensing Symposium, 1318-1321 (2007).

[21] Gilks, W. R., Richardson, S., \& Spiegelhalter, D. J. (1996). Introducing Markov chain Monte Carlo. In W. R. Gilks, S. Richardson, \& D. J. Spiegelhalter (Eds.), Markov Chain Monte Carlo in Practice. London: Chapman \& Hall.

[22] Zhao, Q., Li, Y., Liu, Z., "SAR Image Segmentation Using Voronoi Tessellation and Bayesian Inference Applied to Dark Spot Feature Extraction”, Sensors, 13(11), 14484-14499 (2013).

[23] Oscar Nestares, O., Navarro, R., Portilla, J., Tabernero, A., "Efficient spatial-domain implementation of a multiscale image representation based on Gabor functions", J. Electron. Imaging. 7(1), 166-173 (1998).

[24] Cyganek, B., Siebert, J. P., John Wiley \& Sons, "An introduction to 3d Computer vision techniques and algorithms", (2009).

[25] Duda, R.O., Hart, P. E., "Use of the Hough transformation to detect lines and curves in pictures. Commun”, 15(1), 11-15 (1972).

[26] Michael Laszlo, Sumitra Mukherjee, "A genetic algorithm that exchanges neighbouring centers for k-means clustering”, Pattern Recognition Letters, 28(16), 2359-2366 (2007).

[27] Tang, H., Achary, X. T., Ajoy, A.K., Image Processing: Principles and Applications, 63-78 (2005).

[28] Hou, X., Zhang, L., "Saliency Detection: A Spectral Residual Approach," 2007 IEEE Conference on Computer Vision and Pattern Recognition, 1-8 (2007). 